First published in The Pharmaceutical Journal December 2016 online, DOI:

10.1211/PJ.2017.20202086. Available at: http://www.pharmaceutical-

journal.com/opinion/correspondence/multidisciplinary-preregistration-training-is-the-way-

forward/20202086.article". 


\section{Multidisciplinary preregistration training is the way forward}

Pharmacists increasingly need to work within a multidisciplinary team, especially in light of their increased potential roles. However, there is currently no formalised training model for multidisciplinary learning (MDL) after graduation. Opportunities need to be sought for learning together through provision of placements and learning events. Pilots are currently being carried out to facilitate pre-registration pharmacist training in GP surgeries and trainee doctors working in pharmacies and vice versa. Such schemes need to become commonplace and be extended to learn about other professions in multiple settings. To facilitate this, pharmacists need to identify opportunities and work closely with other local healthcare professionals.

A workshop was organised by Health Education England to develop recognition of MDL benefits. A total of 28 pharmacist pre-registration tutors and 20 colleagues from other disciplines, including nurses, doctors and midwives in London, attended the workshop, split over two events, held in April 2016. Just over half of the attendees had previously taken part in multidisciplinary learning, showing more work needs to be done to work together and understand each other's roles. After the workshop, there was increased knowledge of each professional's role and increased confidence in implementing MDL in practice after the event. Training together in an MDL environment was seen as positive, with no hierarchy seen between professions.

Overall, the attendees supported the implementation of multidisciplinary working in practice, with focus on placement opportunities for trainees. All professions identified activities for other trainees to be involved in, and recognised how working together can achieve greater patient care. Case studies and sharing the potential benefits of MDL with teams were seen as supporters of implementation. Pre-registration training is a key opportunity for MDL implementation to enhance future multidisciplinary working relationships. To enable this, sessions need to be designed to share the vision and importance of such learning for the future of the profession and to inform a strategy for implementation. Furthermore, opportunities need to be created to facilitate and organise MDL at pre-registration level because this was seen as essential for improving patient care. This can be enhanced by building case studies and a structured placement programme.

Investment is needed to support MDL initiatives during pharmacist pre-registration training and beyond to ensure integrated patient care.

Ricarda Micallef

Senior Lecturer

$\underline{\text { r.micallef@kingston.ac.uk }}$

Reem Kayyali 
Associate Professor

Kingston University

Atif Shamim

Lead Pharmacist, Community and Primary Care, London and the South East

Health Education England 